\title{
THE WEAK LEFSCHETZ PRINCIPLE IS FALSE FOR AMPLE CONES*
}

\author{
BRENDAN HASSETT ${ }^{\dagger}$, HUI-WEN LIN ${ }^{\ddagger}$, AND CHIN-LUNG WANG ${ }^{\S}$
}

1. Introduction. Let $X$ be an $(n+1)$-dimensional smooth complex projective variety and let $D$ be a smooth ample divisor of $X$ with inclusion map $i: D \rightarrow X$. The well-known Weak Lefschetz Theorem (see [GrHa]) asserts that the restriction map $i^{*}: H^{k}(X ; \mathbb{Z}) \rightarrow H^{k}(D ; \mathbb{Z})$ is an isomorphism for $k \leq n-1$ and is compatible with the Hodge decomposition. For $n \geq 3$ one deduces from these results that $i^{*}: \operatorname{Pic}(X) \rightarrow$ $\operatorname{Pic}(D)$ is also an isomorphism. Grothendieck has shown that this statement is true over any algebraically closed field [Hart]. While an ample line bundle on $X$ always restricts to an ample line bundle on $D$, it is not at all clear whether $\operatorname{Amp}(D) \subset$ $i^{*} \operatorname{Amp}(X)$, i.e., whether the Weak Lefschetz Principle holds for the ample cone.

In this note we provide two examples showing that the Weak Lefschetz Principle for the ample cone fails in general. One is obtained by blowing up (§2) and the other is a product with a $\mathbb{P}^{1}$ factor $(\S 3)$. We also provide some partial positive results $(\S 4)$. However, a complete picture of how the ample cone behaves under the Weak Lefschetz isomorphism remains elusive.

\section{A blow-up example.}

2.1. The construction. We construct our first counterexample $(X, D)$. Let $\phi: X \rightarrow \mathbb{P}^{4}$ be the blow-up of $\mathbb{P}^{4}$ at two distinct points $p_{1}$ and $p_{2}$. Let $l^{\prime}$ be the line spanned by $p_{1}$ and $p_{2}$ and $D^{\prime}$ a general smooth cubic hypersurface (threefold) containing $p_{1}$ and $p_{2}$ but not the line $l^{\prime}$. The conditions satisfied by $D^{\prime}$ will be made precise in 2.5. We take $D$ to be the proper transform of $D^{\prime}$ in $X$. We will see that $D$ is a very ample divisor in $X$ but $i^{*} \operatorname{Amp}(X) \neq \operatorname{Amp}(D)$. More precisely, $\operatorname{Amp}(D)$ is strictly larger than $i^{*} \operatorname{Amp}(X)$ if and only if the Mori cone (the closure of the real cone generated by numerically equivalent classes of effective one-cycles) $\overline{\mathrm{NE}}(X)$ is strictly larger than $i_{*} \overline{\mathrm{NE}}(D)$ (by Kleiman's criterion for ampleness [Hart]). Let $l$ be the proper transform of $l^{\prime}$ in $X$, which is an effective one-cycle in $X$. By Weak Lefschetz, $l=i_{*} \lambda$ for some one-cycle $\lambda$ on $D$. Our main task is to show that $\lambda \notin \overline{\mathrm{NE}}(D)$. In fact we will determine both the ample cones and the Mori cones of $X$ and $D$.

2.2. Ample and Mori cones of $X$. We first set up some notation; we shall often use a single letter to denote a subvariety and its homology class. Let $E_{1}$ and $E_{2}$ be the exceptional divisors in $X, H^{\prime}$ the general hyperplane in $\mathbb{P}^{4}$ containing $p_{1}$ and $p_{2}$, and $H$ its proper transform in $X$. Note that $l^{\prime}=H^{\prime 3}$ and $H=\phi^{*} H^{\prime}-E_{1}-E_{2}$. It is clear that $\operatorname{Pic}(X)=\mathbb{Z} H+\mathbb{Z} E_{1}+\mathbb{Z} E_{2}$. Since $E_{i} \cong \mathbb{P}^{3}$ and $-\left.E_{i}\right|_{E_{i}}$ is its hyperplane class, $E_{i}^{3}$ corresponds to the class of a line in $E_{i}$. The group of one-cycles (modulo rational or numerical equivalence) is then given by $N(X)=\mathbb{Z} \phi^{*} H^{\prime 3}+\mathbb{Z} E_{1}^{3}+\mathbb{Z} E_{2}^{3}$

${ }^{*}$ Received August 13, 2001; accepted for publication September 15, 2001.

${ }^{\dagger}$ Mathematics Department, Rice University, Houston, USA (hassett@math.rice.edu). The author was partially supported by the Institute of Mathematical Sciences of the Chinese University of Hong Kong and NSF Grant 0070537. He would like to thank the National Center for Theoretical Sciences of Hsinchu, Taiwan, for the invitation that made this collaboration possible.

$\ddagger$ Department of Mathematics, National Central University, Chungli, Taiwan (linhw@math.ncu.edu.tw).

$\S$ Department of Mathematics, National Tsing-Hua University, Hsinchu, Taiwan (dragon@math2.math.nthu.edu.tw). 
and $l=\phi^{*} H^{\prime 3}-E_{1}^{3}-E_{2}^{3}$. As we blow up at only two points, it is readily seen that $\mathrm{NE}(X)=\mathbb{R}_{\geq 0} l+\mathbb{R}_{\geq 0} E_{1}^{3}+\mathbb{R}_{\geq 0} E_{2}^{3}$ which is already a rational closed cone. For later use, we also observe that $l . E_{i}=1$ and $E_{i}^{4}=\left(\left.E_{i}\right|_{E_{i}}\right)^{3}=-1$.

Proposition 2.1. The ample cone $\operatorname{Amp}(X)$ is the interior of the cone generated by $H+E_{1}+E_{2}, H+E_{1}$ and $H+E_{2}$. That is, a $\mathbb{Q}$-divisor $L=\phi^{*} H^{\prime}-a_{1} E_{1}-a_{2} E_{2}$ is ample if and only if $a_{i}>0$ and $a_{1}+a_{2}<1$. Moreover, all ample divisors are automatically very ample.

Proof. By Kleiman's criterion, $L$ is ample if and only if $L . l=1-a_{1}-a_{2}>0$ and $L . E_{i}^{3}=a_{i}>0$.

For the last statement, one observes that $X$ is naturally a smooth toric variety and for smooth toric varieties, ample divisors are automatically very ample (Theorem of Demazure, see e.g. [Oda]).

Corollary 2.2. The divisor $D \sim 3 \phi^{*} H^{\prime}-E_{1}-E_{2}$ is very ample in $X$ but $-K_{X} \sim 5 \phi^{*} H^{\prime}-3 E_{1}-3 E_{2}$ is not even ample. In particular, $X$ is not Fano.

2.3. The main argument. We recall the following criterion for when the blowup of a cubic surface at two points is a Del Pezzo surface:

Proposition 2.3. Let $S^{\prime}$ be a smooth cubic surface containing distinct points $p_{1}$ and $p_{2}, l^{\prime}$ the line spanned by these points, and $S$ the blow-up of $S^{\prime}$ at $p_{1}$ and $p_{2}$. Assume that $l^{\prime} \cap S^{\prime}$ consists of three distinct points, and no line containing any one of these three points is contained in $S^{\prime}$. Then $S$ is a Del Pezzo surface.

Proof. This argument is inspired by some remarks of Naruki [Nar]. We write $l^{\prime} \cap S^{\prime}=\left\{p_{1}, p_{2}, p_{3}\right\}, \tau^{\prime}: T=\operatorname{Bl}\left(S^{\prime}\right)_{p_{1}, p_{2}, p_{3}} \rightarrow S^{\prime}$, and $\tau: T \rightarrow S$ for the induced map. Let $F_{i} \subset T$ denote the exceptional curve over the point $p_{i}$, so that $K_{T}=$ $\tau^{\prime *} K_{S^{\prime}}+F_{1}+F_{2}+F_{3}=\tau^{*} K_{S}+F_{3}$. Projection from the line $l^{\prime}$ induces a morphism $\mu: T \rightarrow \mathbb{P}^{1}$; the fibers of $\mu$ are intersections of $S^{\prime}$ with hyperplanes containing $l^{\prime}$. In particular, the general fiber is a smooth plane cubic, i.e., $\mu$ is an elliptic fibration. Since $S^{\prime}$ contains no lines containing any of the $p_{i}$, the fibers of $\mu$ are irreducible. Furthermore, the hyperplane class of $\mathbb{P}^{1}$ pulls back to $-K_{T}=-\tau^{*} K_{S}-F_{3}$.

To prove that $-K_{S}$ is ample we apply the Nakai-Moishezon criterion: $-K_{S}$ is ample provided that $K_{S}^{2}>0$ and $-K_{S} C>0$ for each closed irreducible curve $C \subset S$. It suffices to check the proper transform $C_{T}$ of $C$ intersects $-\tau^{*} K_{S}$ positively. If $\mu$ contracts $C_{T}$ to a point then $\left(-\tau^{*} K_{S}\right) C_{T}=F_{3} C_{T}>0$ because the fibers of $\mu$ are irreducible and $F_{3}$ is a section of $\mu$. On the other hand, if $C_{T}$ dominates $\mathbb{P}^{1}$ then $-K_{T} C_{T}$ is positive. Since $C_{T} \neq F_{3},\left(-\tau^{*} K_{S}\right) C_{T}$ is positive as well.

REMARK 2.4. The classification theory of surfaces implies that a cubic surface $S^{\prime}$ is the blow-up of $\mathbb{P}^{2}$ at six general points [GrHa]. Precisely, we require that no two of the points coincide, no three are collinear, and no six are contained in a plane conic. Implicit in our proof is a precise condition for when the blow-up of $\mathbb{P}^{2}$ at eight general points is a Del Pezzo surface. In addition to the conditions listed above, we require that there exists no plane cubic passing through all eight points and singular at one of the eight. Equivalently, the pencil of cubic curves passing through the eight points should have reduced base locus. This criterion was suggested without proof in §26 of [Manin].

Assumptions 2.5. We make the following generality assumptions on $D^{\prime}$ :

1.The intersection $l^{\prime} \cap D^{\prime}$ contains three distinct points.

2.There are a finite number of lines contained in $D^{\prime}$ and meeting $l^{\prime} \cap D^{\prime}$. 
For a general point of a cubic threefold, there are six lines containing the point and contained in the threefold.

Let $S^{\prime}=D^{\prime} \cap H^{\prime}$ be a hyperplane section of $D^{\prime}$ containing $p_{1}$ and $p_{2}$, a cubic surface in $H^{\prime} \cong \mathbb{P}^{3}$. We choose $S^{\prime}$ so that it is smooth and does not contain any lines meeting $l^{\prime} \cap D^{\prime}$ (such an $S^{\prime}$ exists by our second assumption above.) Let $S \subset H$ be its proper transform, the blow-up of $S^{\prime}$ at $p_{1}$ and $p_{2}$.

Note that $S=\left.\left(\phi^{*} H^{\prime}-E_{1}-E_{2}\right)\right|_{D}$ and $K_{D}=\left.\left(-2 \phi^{*} H^{\prime}+2 E_{1}+2 E_{2}\right)\right|_{D}=$ $-2 S$. Also, since $S^{\prime}$ is a cubic surface inside $H^{\prime} \cong \mathbb{P}^{3}$, adjunction shows that $K_{S^{\prime}}=$ $\left.\left(K_{H^{\prime}}+S^{\prime}\right)\right|_{S^{\prime}}=\left.\left(-\left.4 H^{\prime}\right|_{H^{\prime}}+\left.3 H^{\prime}\right|_{H^{\prime}}\right)\right|_{S^{\prime}}=-\left.H^{\prime}\right|_{S^{\prime}}$. By the blow-up formula, $K_{S}=$ $\phi^{*} K_{S^{\prime}}+\left.E_{1}\right|_{S}+\left.E_{2}\right|_{S}=\left.\left(-\phi^{*} H^{\prime}+E_{1}+E_{2}\right)\right|_{S}=-\left.H\right|_{S}$.

Proposition 2.6. Consider an effective one-cycle $\tilde{l}:=\phi^{*}{H^{\prime}}^{3}-a_{1} E_{1}^{3}-a_{2} E_{2}^{3}$ in $X$ with $a_{i} \leq 1$ and $a_{1}+a_{2}>1$. Let $\tilde{\lambda}$ be the one-cycle class in $D$ which corresponds to $\tilde{l}$. Then $\tilde{\tilde{\lambda}} \notin \overline{\mathrm{NE}}(D)$. In particular $\lambda \notin \overline{\mathrm{NE}}(D)$.

Proof. If $\tilde{\lambda} \in \overline{\mathrm{NE}}(D)$ then $\tilde{\lambda}$ is the limit of a sequence $\left\{C_{i}\right\}$ of effective one-cycles on $D$ with rational coefficients. By assumption, $\tilde{\lambda} . S=1-a_{1}-a_{2}<0$ which implies that $C_{i} . S<0$ for $i$ large enough. In particular this shows that $C_{i} \subset S$ for $i$ large enough.

On the other hand, the sequence $C_{i} \cdot K_{S}$ has limit $\tilde{l} \cdot(-H)=-1+a_{1}+a_{2}>0$. This imples that $C_{i} . K_{S}>0$ for $i$ large enough, contradicting the fact that $-K_{S}$ is an ample divisor in $S$.

Remark 2.7. To get counterexamples to the Weak Lefschetz Principle, it is essential that the cubic threefold $D^{\prime}$ does not contain $l^{\prime}$. In fact, if $D^{\prime}$ contains $l^{\prime}$ then $S^{\prime}$ will also contain $l^{\prime}$. Thus $S$ is not a blow-up of $\mathbb{P}^{2}$ at eight 'general' points, $-K_{S}$ is not ample, and no contradiction arises.

2.4. Ample and Mori cones of $D$. We retain the assumptions of section 2.3. Proposition 2.6 exhibits effective classes in $X$ that are not represented by effective classes in $D$. We would like to show here that all other effective classes in $X$ lie in the image $i_{*} \mathrm{NE}(D)$.

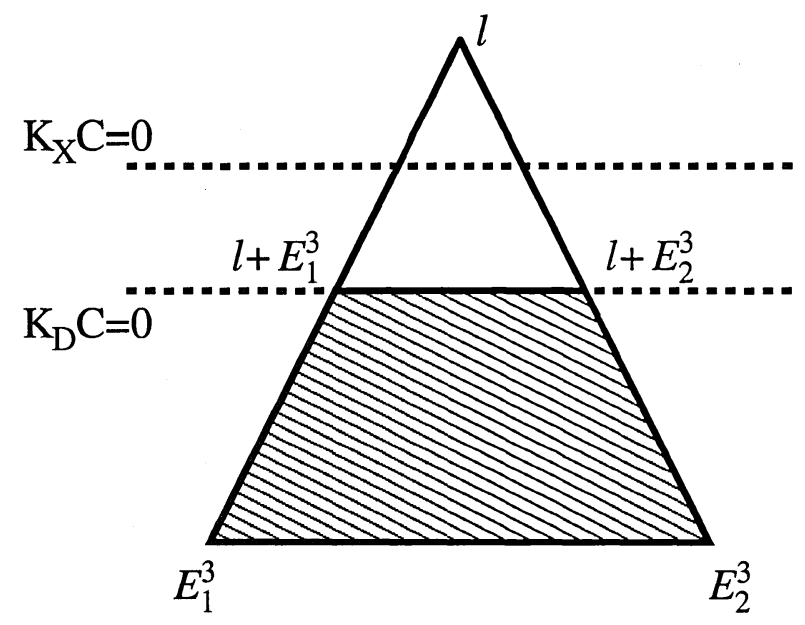

FIG. 2.1. Mori cones of $X$ and $D$

THEOREM 2.8. The image of the Mori cone $i_{*} \mathrm{NE}(D)$ is a closed rational polyhedral cone generated by $l+E_{1}^{3}, l+E_{2}^{3}, E_{1}^{3}$ and $E_{2}^{3}$. The image of the ample cone 
$i_{*} \operatorname{Amp}(D)$ is the interior of the rational polyhedral cone generated by $H, H+E_{1}$, $H+E_{2}$ and $H+E_{1}+E_{2}$ (the nef cone).

Proof. Recall that the class $l+E_{1}^{3}=\phi^{*} H^{\prime 3}-E_{2}^{3}$ is represented by the proper transform of a line in $\mathbb{P}^{4}$ which contains $p_{2}$ and is distinct from $\ell$. Since $D^{\prime}$ is a cubic threefold, through every point of $D^{\prime}$ one may find lines [Harris]. The proper transform in $D$ of one such line will have the class $l+E_{1}^{3}$. The same argument applies to $l+E_{2}^{3}$. Since the cone generated by $l+E_{1}^{3}, l+E_{2}^{3}, E_{1}^{3}$ and $E_{2}^{3}$ is precisely $\mathrm{NE}(X)$ with the classes considered in Proposition 2.6 removed, we conclude that it is $i_{*} \mathrm{NE}(D)$.

The statement on the ample cone is an application of Kleiman's criterion to $L:=H+a_{1} E_{1}+a_{2} E_{2}$; intersecting $L$ with $l+E_{1}^{3}, l+E_{2}^{3}, E_{1}^{3}$ and $E_{2}^{3}$, we get $0<a_{1}, a_{2}<1$.

REMARK 2.9. Szendroi [Sz] has constructed examples in a similar vein, as counterexamples to conjectures of Cox and Katz on the ample cones of anticanonical hypersurfaces in toric varieties.

3. A product example with $\mathbb{P}^{1}$ factors. Much simpler examples can be found if $X$ is a product. Take $X=\mathbb{P}^{1} \times \mathbb{P}^{d}$ (for $d \geq 3$ ) and let $D$ be a divisor of type $(d, b)$ with $b \in \mathbb{N}$. The divisor $D$ is very ample and thus a generic member of $|D|$ is smooth. It has defining equation

$$
x^{d} f_{0}+x^{d-1} y f_{1}+\ldots+f_{d} y^{d}=0
$$

where $x, y$ are coordinates of $\mathbb{P}^{1}$ and the $f_{i}$ 's are polynomials of degree $b$ in $\mathbb{P}^{d}$. The projection $p: D \rightarrow \mathbb{P}^{d}$ has positive dimensional fibers exactly when $f_{0}=f_{1}=\ldots=$ $f_{d}=0$. This has no nontrivial solutions for general $f_{i}$ 's since there are more equations than variables. However, if $p$ is a finite morphism then each ample divisor $L$ on $\mathbb{P}^{d}$ pulls back to an ample divisor on $D$ (this follows from either the Nakai-Moishezon criterion or Kleiman's criterion), yet this pull-back divisor is the restriction of the divisor $\mathbb{P}^{1} \times L$ on $X=\mathbb{P}^{1} \times \mathbb{P}^{d}$, which is evidently not ample. This gives another (easier) counterexample to the Weak Lefschetz Principle.

The presence of a one-dimensional factor here is crucial (cf. Theorem 4.1).

\section{Positive results.}

4.1. Products. It is trivial that the Weak Lefschetz Principle holds if $X$ has Néron-Severi rank one and $\operatorname{dim} X \geq 4$. One may generalize this in a straightforward manner to obtain:

TheOREM 4.1. Let $i: D \rightarrow X=\prod X_{j}$ be a smooth ample divisor in a finite product of smooth projective varieties, each with dimension $\geq 2$ and Néron-Severi rank equal to one. Assume that $\operatorname{dim} X \geq 4$ and $\operatorname{Pic}(X)=\bigoplus p_{j}^{*} \operatorname{Pic}\left(X_{j}\right)$, where $p_{j}: X \rightarrow X_{j}$ is the projection map. Then $i^{*} \operatorname{Amp}(X)=\operatorname{Amp}(D)$.

Proof. Let $H_{j}$ be an ample class on $X_{j}$ and let $h_{j}=p_{j}^{*} H_{j}$. Notice the following fact: If $n_{j}=\operatorname{dim} X_{j}$, then $\prod h_{j}^{m_{j}}$ is an effective cycle if and only if $m_{j} \leq n_{j}$ for all $j$ and $p=\prod h_{j}^{n_{j}}$ is a positive integer. It is then easy to see that $\sum a_{j} h_{j}$ is an ample class on $X$ if and only if that $a_{j}>0$ for all $j$ : simply intersect it with $h_{j}^{n_{j}-1} \cdot \prod_{k \neq j} h_{k}^{n_{k}}$ to get $a_{j} p>0$.

Now let $D$ be given by the ample class $\sum d_{j} h_{j}$ with $d_{j}>0$. Since $\operatorname{dim} X \geq 4$, a divisor on $D$ takes the form $\left.L\right|_{D}=\left.\sum a_{j} h_{j}\right|_{D}$. We need to show that $\left.L\right|_{D}$ is ample 
implies that $a_{j}>0$ for all $j$. Indeed, since $n_{j} \geq 2$, we simply intersect $\left.L\right|_{D}$ with the effective cycle $\left.\left.h_{j}\right|_{D} ^{n_{j}-2} \cdot \prod_{k \neq j} h_{k}\right|_{D} ^{n_{k}}$ on $D$ to get

$$
0<h_{j}^{n_{j}-2} \cdot \prod_{k \neq j} h_{k}^{n_{k}} \cdot \sum a_{j} h_{j} \cdot \sum d_{j} h_{j}=a_{j} d_{j} p .
$$

It follows that $a_{j}>0$.

REMARK 4.2. The condition $\operatorname{Pic}(X)=\bigoplus p_{j}^{*} \operatorname{Pic}\left(X_{j}\right)$ holds if all but one of the factors satisfy $h^{1}\left(X_{j}, \mathcal{O}_{X_{j}}\right)=0$, or more generally, if the Jacobians of the factors admit no nontrivial endomorphisms $\eta: \operatorname{Jac}\left(X_{i}\right) \rightarrow \operatorname{Jac}\left(X_{j}\right), i \neq j$.

4.2. A partial theorem on Mori cones. The counterexample of section 2 suggests the following equality on the negative part of Mori cones of $D$ and $X$ (compare Proposition 2.1 and Theorem 2.8). The proof relies on Mori's theory of extremal rays [Mori] and is essentially contained in [Wiś] and [Kollár].

THEOREM 4.3. Let $i: D \rightarrow X$ be a smooth ample divisor in a smooth variety $X$ with $\operatorname{dim} X \geq 4$. Then $i_{*} \mathrm{NE}(D)_{K_{D} \leq 0}=\mathrm{NE}(X)_{K_{D} \leq 0}$.

Proof. Since $K_{D}=\left.K_{X}\right|_{D}+\left.D\right|_{D}$, which is numerically strictly more positive than $K_{X}$, we know by Mori's theory that $\mathrm{NE}(X)_{K_{D} \leq 0}$ is a finite polyhedral cone generated by extremal rays. Let $R=\mathbb{R} C$ with $C \cong \mathbb{P}^{1}$ be such a ray and $\phi: X \rightarrow Y$ the corresponding contraction. We want to show that the class of $C$ is also an effective class in $D$. If $\phi$ has a fiber $F$ of dimension at least two then $D \cap F$ has positive dimension and contains a curve with class in $R$. If all fibers are one-dimensional, Wiśniewski's theorem shows that $Y$ is smooth and either $\phi$ is a blow-up of $Y$ along a smooth codimension-two subvariety $Z$ or $\phi$ is in fact a conic bundle. These cases are ruled out when $\operatorname{dim} X \geq 4$ by an argument of Kollár (the Lemma of [Kollár]).

\section{REFERENCES}

[GrHa] P. Griffiths AND J. HARRIS, Principles of Algebraic Geometry, Wiley-Interscience, New York, 1978.

[Harris] J. HARRIS, Algebraic Geometry: A First Course, Graduate Texts in Math., 133 (1992), Springer-Verlag, New York.

[Hart] R. HARTSHORne, Ample Subvarieties of Algebraic Varieties, Lecture Notes in Math., 156 (1970), Springer-Verlag, Berlin-New York.

[Kollár] J. Kollár, Appendix to "C. Borcea; Homogeneous Vector bundles and families of Calabi-Yau Threefolds, II", Proc. Symp. Pure Math. 52 (1991).

[Manin] Y.I. Manin, Cubic Forms: Algebra, Geometry, Arithmetic, North-Holland, Amsterdam, 1974.

[Mori] S. MoRI, Threefolds whose canonical bundles are not numerically effective, Ann. of Math. 116 (1982), pp. 133-176.

[Nar] I. NARUKI, Cross ratios as moduli of del Pezzo surfaces of degree one, Proc. Japan Acad. Ser. A Mat. Sci., 56 (1980), no. 7, pp. 328-331.

[Oda] T. OdA, Convex Bodies and Algebraic Geometry, Springer-Verlag, Berlin, 1988.

[Sz] B. SzENDROI, On a conjecture of Cox and Katz, to appear in Math. Z.

[Wiś] J.A. WIśnIEWSKI, On contractions of extremal rays of Fano manifolds, J. reine angew. Math. 417 (1991), pp. 141-157. 
